\title{
Roles of MYC-targeting long non-coding RNA MINCR in cell cycle regulation and apoptosis in non-small cell lung Cancer
}

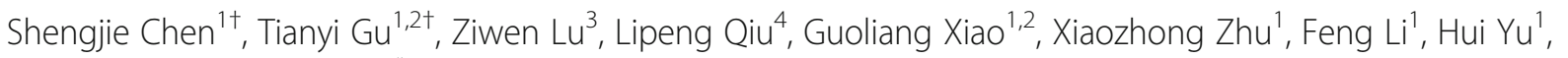
Gang Li ${ }^{1}$ and Hanqing $\mathrm{Liu}^{3^{*}}$ (D)

\begin{abstract}
Background: Non-small cell lung cancer (NSCLC) is one of the leading causes of cancer death in the world, and has a relatively low survival rate. Long non-coding RNAs (IncRNAs) have been demonstrated to modulate cancer progression through a variety of molecular mechanisms. We sought to investigate the role and potential mechanism of MYC-induced long non-coding RNA (MINCR) in NSCLC.

Methods: Expression levels of MINCR was first identified using The Cancer Genome Atlas (TCGA), further confirmed with specimens from 29 NSCLC patients and three cell lines using qRT-PCR. Overexpression and knockdown of MINCR were performed in NSCLC cell lines through MINCR overexpression vectors and synthesized siRNAs, respectively. The roles of MINCR in NSCLC cell lines, such as cell proliferation, cell cycle arrest, and apoptosis, were identified by MTT, flow cytometry, and Western blot. The modulation of MINCR-regulated genes, including c-Myc and its downstream effectors, as well as apoptosis-associated genes, was analyzed using Western blot.

Results: MINCR expression was increased in NSCLC patients from TCGA datasets, and was also significantly increased in our collected specimens from NSCLC patients and NSCLC cell lines. Knocking down of MINCR greatly inhibited the growth of NSCLC cell lines PC9 and A549. In addition, silencing of MINCR induced cell cycle arrest and apoptosis. Furthermore, silencing of MINCR reduced the expression levels of oncogene c-Myc and its downstream cyclin A, cyclin D, CD4, and CDK2, as well as apoptosis-associated Bcl-2, while significantly increased the expression levels of cleaved PARP-1. In the meantime, overexpression of MINCR remarkably enhanced cell proliferation of PC9 cells and activated c-Myc and its downstream effectors.
\end{abstract}

Conclusion: MINCR exerted inhibitory effects on the cell cycle arrest and apoptosis of NSCLC cells by activating cMyc and its downstream effectors, suggesting that this IncRNA could be used as a potential therapeutic target for the treatment of NSCLC.

Keywords: Long non-coding RNA, MINCR, Non-small cell lung cancer, C-Myc

\section{Introduction}

Lung cancer is one of the leading causes of malignancy induced human death. Non-small cell lung cancer (NSCLC) is a major type of lung cancer, accounting for $80 \%$ of all cases of lung cancers. Despite some effective progresses has been made in chemotherapy and targeted

\footnotetext{
* Correspondence: hanqing@ujs.edu.cn

+Shengjie Chen and Tianyi Gu contributed equally to this work.

${ }^{3}$ School of Pharmacy, Jiangsu University, Zhenjiang 212013, Jiangsu Province, China

Full list of author information is available at the end of the article
}

molecular therapies, the 5-year survival rate of lung cancer remains low, ranging from 10 to $30 \%$ all over the world. Thus, it is critically important to elucidate the underlying molecular mechanisms of NSCLC to develop noval therapeutic drugs.

Over the past decade, the development in deep sequencing of mammalian transcriptomes has led to the discovery of more than 100,000 non-coding RNAs [1,2]. Sharing certain structural similarities with protein-coding mRNAs, long non-coding RNAs (lncRNAs) refer to transcripts that are longer than 200 nucleotides but

(C) The Author(s). 2019 Open Access This article is distributed under the terms of the Creative Commons Attribution 4.0 International License (http://creativecommons.org/licenses/by/4.0/), which permits unrestricted use, distribution, and 
without protein-coding potential [2-4]. It has been revealed that IncRNAs are very heterogeneous in their mechanisms of function. Therefore, without any surprise, as the researches go on, IncRNAs have been demonstrated to exhibit versatile functions in diverse biological processes [5-8]. More importantly, recent studies showed that lncRNAs are involved in tumorigenesis and development of many kinds of cancers [9-12].

About 3 years ago, Doose et al. discovered that MYC-induced lncRNA (MINCR) was able to modulate the transcriptional network of MYC (c-Myc) in Burkitt lymphoma cells [13]. After that, MINCR was found to be significantly increased, and play an oncogenic role in cancers, such as gallbladder cancer and hepatocellular carcinoma [14, 15]. Wang et al. revealed that MINCR promotes gallbladder cancer progression in part by sponging miR-26a-5p and activating enhancer of zeste homolog 2 (EZH2) signaling; while Cao et al. reported that MINCR enhances the proliferation, migration, and invasion of hepatocellular carcinoma cells $[14,15]$. All these studies imply that MINCR could be a therapeutic target as well as prognostic marker for cancer treatment.

As we are interested in the treatment of NSCLC, we screened a panel of lncRNAs, and found that MINCR was highly expressed in patient samples and cell lines of NSCLC. In the current study, we evaluated the function of MINCR in the proliferation and apoptosis of NSCLC cell lines in vitro, and then investigated the impact of MINCR on oncogene c-Myc and its downstream effectors, as well as apoptosis-associated genes to reveal the underlying mechanism beneath these phenomena.

\section{Materials and methods}

Data collection from the Cancer genome atlas (TCGA)

The expression of MINCR in two subtypes of NSCLC, including lung adenocarcinoma (LUAD) and lung squamous carcinoma (LUSC), were extracted from TCGA. For LUAD, The expression of MINCR was collected from 526 tumor and 59 non-tumor samples; for LUSC, the expression of MINCR was collected from 501 tumor and 49 non-tumor samples.

\section{Specimens and ethics statement}

Twenty-nine new NSCLC patients who have not received any anti-cancer radio- or chemotherapy were enrolled in this study. After surgical processes in Affiliated Hospital of Jiangsu university, their paired tissue specimens (NSCLC tissue and para-tumor tissue) were immediately preserved in RNA-fixer reagent (Bioteke, Beijing, China), and frozen at $-80^{\circ} \mathrm{C}$ until use. All these specimens were pathologically confirmed by two experienced pathologists. Clinicopathological
Table 1 Clinicopathological characteristics of NSCLC patients

\begin{tabular}{|c|c|c|}
\hline Characteristics & Number $(n=29)$ & Percent (\%) \\
\hline \multicolumn{3}{|l|}{ Age (years) } \\
\hline$<60$ & 10 & 34.5 \\
\hline$\geq 60$ & 19 & 65.5 \\
\hline \multicolumn{3}{|l|}{ Gender } \\
\hline Male & 17 & 58.6 \\
\hline Female & 12 & 41.4 \\
\hline \multicolumn{3}{|l|}{ History of Smoking } \\
\hline Never & 18 & 62.1 \\
\hline Current & 11 & 37.9 \\
\hline \multicolumn{3}{|l|}{ Tumor size (cm) } \\
\hline $3 \mathrm{~cm}$ or less & 16 & 55.2 \\
\hline over $3 \mathrm{~cm}$ & 13 & 44.8 \\
\hline \multicolumn{3}{|l|}{ Histological grade } \\
\hline High & 3 & 10.3 \\
\hline High to middle & 3 & 10.3 \\
\hline Middle & 5 & 17.2 \\
\hline Middle to low & 6 & 20.7 \\
\hline Low & 2 & 6.9 \\
\hline Other & 10 & 34.5 \\
\hline \multicolumn{3}{|l|}{ Histological classification } \\
\hline Adenocarcinoma & 21 & 72.4 \\
\hline Squamous cell carcinoma & 6 & 20.7 \\
\hline Adenosquamous carcinoma & 2 & 6.9 \\
\hline \multicolumn{3}{|l|}{ Lymph node metastasis } \\
\hline Yes & 5 & 17.2 \\
\hline No & 24 & 82.8 \\
\hline
\end{tabular}

characteristics of these NSCLC patients are summarized in Table 1.

This study was approved by the Human Research Ethics Committee of Jiangsu University. All the subjects have signed informed consent before the surgery.

\section{Antibodies and reagents}

The antibodies for the Western blot were c-Myc (1: 1000, Cell Signaling Technology), cylin-dependent kinase (CDK) 4 (1:500, Santa Cruz), CDK2 (1:500, Santa Cruz), Cyclin A (1:1000, Santa Cruz), Cyclin D (1:1000, Cell Signaling Technology), $\beta$-actin (1: 1000, BEIDI Bio.). Other reagents: TRIzol Reagent (Life Technologies, Grand Island, NY, USA), VFITC/PI staining reagent (Yeasen Biotech, Shanghai, China), BCA Protein Assay Kit (Beyotime Biotech, Nantong, China), and MTT (3-[4]-2, 5-diphenyltetrazolium bromide thiazolyl blue) (Sigma-Aldrich, $\mathrm{St}$ Louis, MO, USA). 


\section{Cell culture}

Human non-small cell lung cancer cell lines (PC9, HCC827, A549) and human normal lung cell line $16 \mathrm{HBE}$ were obtained from the American Type Culture Collection (ATCC, USA). PC9 and HCC827 cells were grown in RPMI1640 (Gibco, Thermo Fisher Scientific, USA); while A549 and 16HBE cells were grown in Dulbecco's modified Eagle's medium (DMEM). Both media were supplemented with $10 \% \mathrm{FBS}, 2 \mathrm{mmol} / \mathrm{L}$ L-glutamine and $100 \mathrm{U} / \mathrm{mL}$ penicillin $/ 100 \mu \mathrm{g} / \mathrm{mL}$ streptomycin (Life Technologies, Grand Island, NY, USA) in a humidified $37^{\circ} \mathrm{C}$ incubator with $5 \% \mathrm{CO}_{2}$.

\section{Cell transfection}

Two small interfering RNAs (siRNAs) against MINCR, si-MINCR-01 and si-MINCR-02, and negative control
siRNA (si-NC) were purchased from Shanghai Gene Pharma Company (China). The siRNA sequences used were as follows: si-MINCR-01 (forward: 5'-GAGCCU UGUUUGCCAUUAATT-3'; reverse: 5' -UUAAUG GCAAACAAGGCUCTT-3'), si-MINCR-02 (forward: 5'-GGGAAGAGUGCGUCUGUGATT-3'; reverse: 5' UCACAGACGCACUCUUCCCTT-3'), and si-NC (forward: 5' - CGUACGCGGAAUACUUCGATT-3'; reverse: 5' - UCGAAGUAUUCCGCGUACGTT-3'). For over-expression studies, the full-length MINCR was synthesized by GeneScript Co. (Nanjing, China), and then cloned into the BamHI and EcoRI sites of pcDNA 3.1(+) expression vector. Transfection was carried out in accordance with the instructions of Lipofectamine 2000 transfection reagent (Invitrogen) [15-17]. The final concentration of MINCR
A

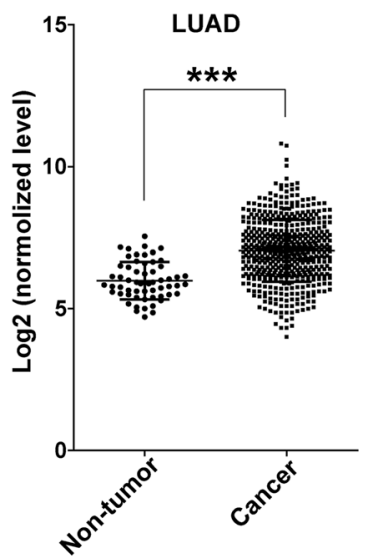

C

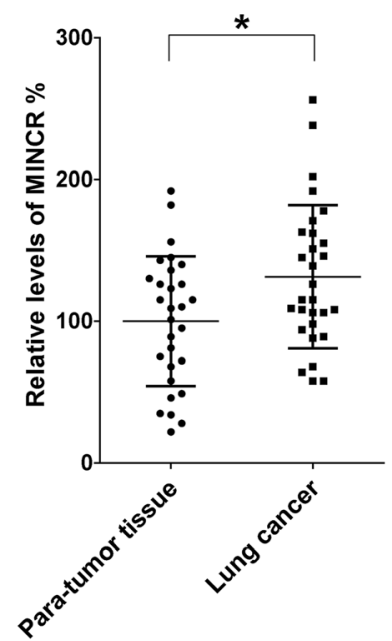

B

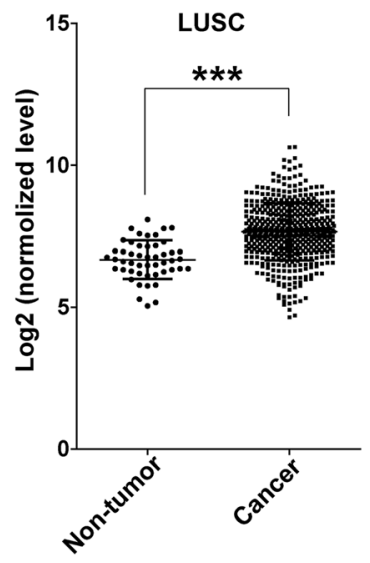

D

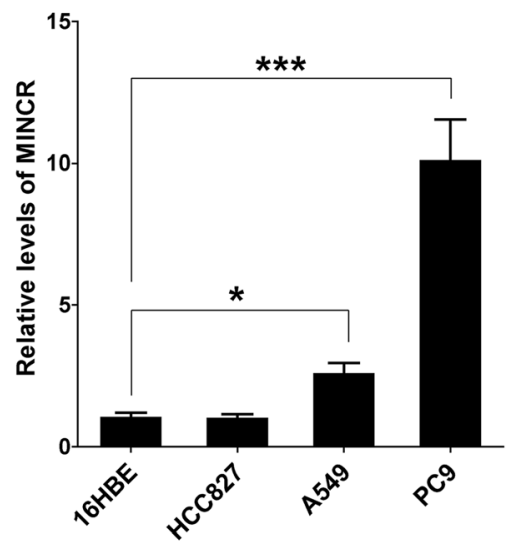

Fig. 1 The expression of MINCR in NSCLC tissues and lung cancer cell lines. a-b, comparing expression of MINCR in non-tumor and NSCLC tissues (Lung Adenocarcinoma, LUAD; Lung Squamous Cell Carcinoma, LUSC) using data from The Cancer Genome Atlas (TCGA). c, relative expression levels of MINCR in para-tumor and cancer tissues from NSCLC patients $(N=29)$. d, relative expression levels of MINCR in $16 \mathrm{HBE}$, HCC827, A549 and PC9 cell lines $(N=3)$. Data were presented as mean \pm standard deviation (SD); ${ }^{*}, p<0.05 ;{ }^{* *}, p<0.01 ;{ }^{* * *}, p<0.001$ 
A

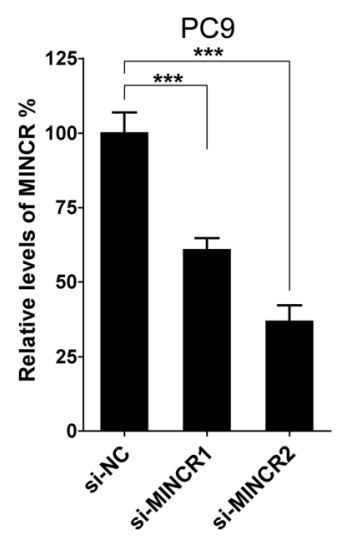

C
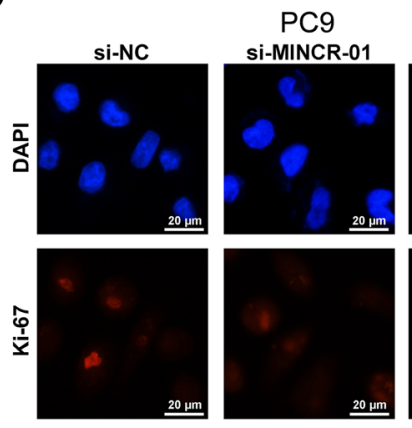

E

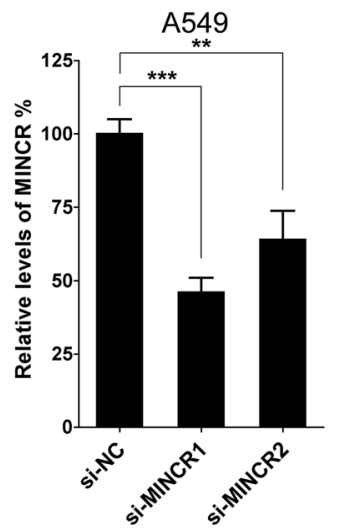

G
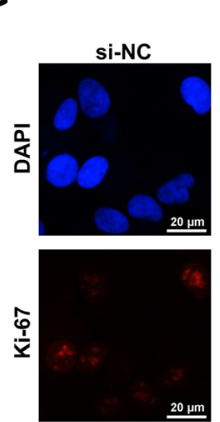

A549

si-MINCR-01

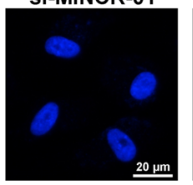

si-MINCR-02
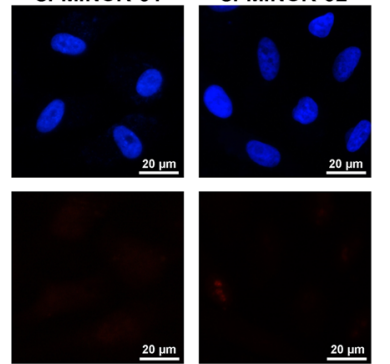

B

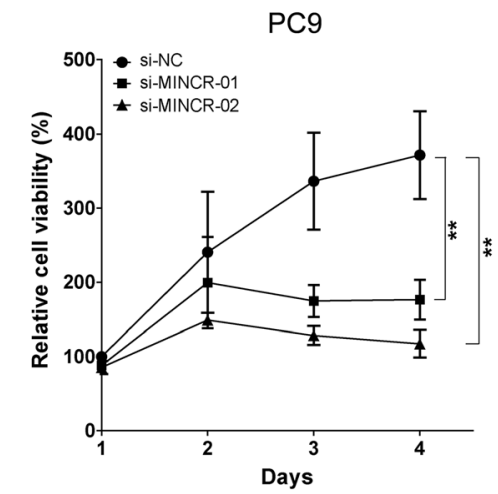

D

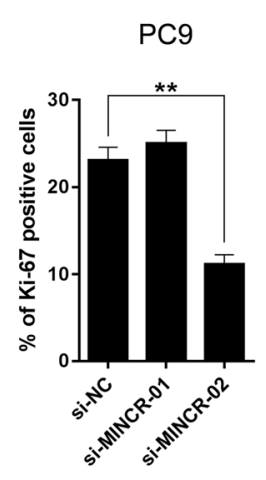

F

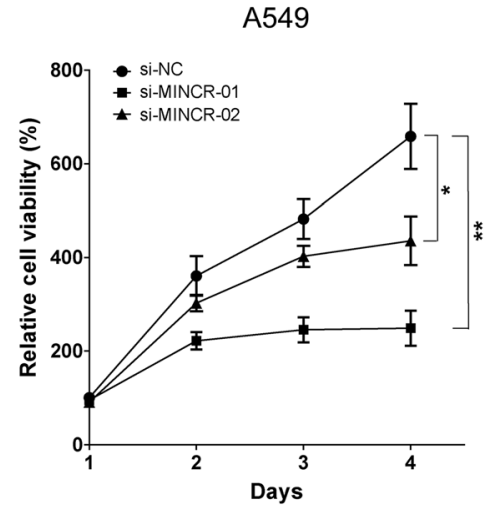

H

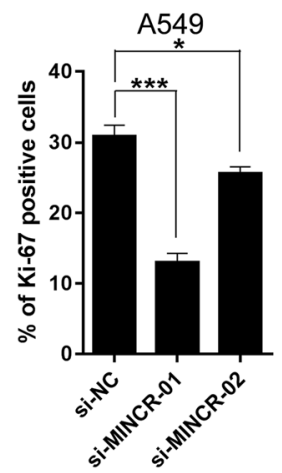

Fig. 2 (See legend on next page.) 
(See figure on previous page.)

Fig. 2 The effect of MINCR silencing on NSCLC cells proliferation. NSCLC cells were transfected with negative control small interfering RNA (siRNA) (si-NC), MINCR siRNA-1 (si-MINCR-01), or MINCR siRNA-2 (si-MINCR-02), respectively, and the viabilities and proliferation were detected using MTT and immunofluorescence. $\mathbf{a}-\mathbf{b}$, silencing of MINCR in PC9 cells (a) and A549 cells (b) using siRNAs. c-e, The corresponding cell viabilities of PC9 cells $(\mathbf{c})$, representative images $(\mathbf{d})$ and statistic graphs $(\mathbf{e})$ of proliferation marker Ki-67 positive cells. f-h, The corresponding cell viabilities of A549 cells (f), representative images $(\mathbf{g})$ and statistic graphs $(\mathbf{h})$ of proliferation marker Ki-67 positive cells. Each experiment was repeated independently at least three times. Data were presented as mean $\pm \mathrm{SD} ;{ }^{*}, p<0.05 ;{ }^{* *}, p<0.01 ;{ }^{* *}, p<0.001$

siRNAs and their negative controls for transfection were $25 \mathrm{nM}$.

\section{Cell viability assay}

Cells were cultured in a 96-well plate with 3000 cells per well. Silencing the MINCR to measure proliferation requires siRNA reverse transfection while seeding cells. Cell viability was measured every $24 \mathrm{~h}$ thereafter for $72 \mathrm{~h}$, and cell viability was measured by the MTT method according to the manufacturer's instructions. Each experiment was repeated independently at least three times $[13,18,19]$.

\section{Immunofluorescence assay}

Immunofluorescence staining was carried out using the method described in several previous studies [19-21]. $72 \mathrm{~h}$ post transfection, cells were fixed in $4 \%$ paraformaldehyde (Sigma-Aldrich, St Louis, MO, USA) for $10 \mathrm{~min}$ and permeabilized by $0.2 \%$ Triton $\mathrm{X}-100$ for $5 \mathrm{~min}$ at room temperature. Fixed cells were washed three times in PBS and blocked for $30 \mathrm{~min}$ with 3\% BSA in PBS and then incubated with an anti-Ki-67 (Santa Cruz, Dallas, TE, USA) antibody for $2 \mathrm{~h}$ at RT, washed three times in PBS and incubated with goat anti-mouse Alexa-fluor 594 secondary antibody (Thermo Scientific, Waltham, MA, USA) for $1 \mathrm{~h}$ at room temperature. Finally, cells were incubated with 4',6-diamidino-2-phenylindole (DAPI) for $5 \mathrm{~min}$ at room temperature. Images were captured using Nikon Eclipse.

\section{Western blot analysis}

The standard procedures for Western blotting experiments in this study were performed according to the procedures given in previous studies $[12,18,22,23]$. Cells were collected $72 \mathrm{~h}$ post transfection, and lysed in Lysis Bufer $(50 \mathrm{mM}$ Tris- $\mathrm{HCl}$ (pH 7.4), $150 \mathrm{mM} \mathrm{NaCl}, 1 \mathrm{mM}$ PMSF, $1 \mathrm{mM}$ EDTA, 1\% Triton $\mathrm{x}-100,1 \%$ Halt Phosphatase Inhibitor Cocktail EDTA-free 100x (Thermo Scientific, Rockford, IL, USA), and $30 \mu \mathrm{g}$ of protein was run over $10 \%$ or $12 \%$ SDS-PAGE gels and eletrophoretically transferred to a PVDF membrane (immobilonPSQ Transfer Membrace, Millpore, Billerica, MA, USA). Then membranes were blocked in $5 \%$ non-fat milk for $1 \mathrm{~h}$ at room temperature, and incubated with specific antibodies at $4{ }^{\circ} \mathrm{C}$ overnight followed by HRP-conjugated secondary antibodies (Jackson Immuno Research, West Grove, PA, USA) at room temperature for one hour. Signals were detected with SuperSignal WestPico Chemiluminescent Substrate (Thermo scientific, Waltham, MA, USA). Each experiment was performed independently at least three times.

\section{Real-time quantitative PCR (qRT-PCR) analysis}

qRT-PCR experiments were performed using the methods described in previous studies. To examine expression of MINCR, total RNAs were collected from NSCLC cells $48 \mathrm{~h}$ post transfection, and isolated using Trizol Reagent. First strand cDNA synthesis kit and AceQ qPCR kit were all from Vazyme (Vazyme Biotech, Nanjing, China). The following primer pairs were used: MINCR (Forward: 5' - GTGTCT GGACACCAGAGGAGT -3'; Reverse: 5'- GGGGCA GAGTCACAAAGC - 3') and GAPDH: (Forward: 5'CAAGGTCATCCATGACAACTTTG - 3'; Reverse: 5' GTCCACCACCCTGTTGCTGTAG -3') [13, 15, 19, 21].

\section{Cell cycle and apoptosis analysis}

Cells were cultured in 6 -cm plates at $1 \times 10^{6}$ cells/dish and cultured for $72 \mathrm{~h}$. Silencing the MINCR to measure cell cycle and apoptosis requires siRNA reverse transfection while seeding cells. Cells were collected and subjected to cell cycle and apoptosis assays as described in several previous studies $[13,18,19]$. For cell cycle analysis, cells were collected and incubated with prechilled $70 \%$ ethanol overnight at $4{ }^{\circ} \mathrm{C}$. Then, cells were washed with prechilled PBS and incubated with propidium iodide (Sigma-Aldrich, St. Louis, MO, USA) at $37^{\circ} \mathrm{C}$ for $1 \mathrm{~h}$. For Apoptosis analysis, cells were collected and incubated with Annexin V-FITC and PI Staining Solution (Yeasen Biotech, Shanghai, China) at room temperature for $30 \mathrm{~min}$ in the dark. Both were detected by BD Flow Cytometer (Franklin Lake, NJ, USA), and analyzed using the FlowJo software (Ashland).

\section{Statistical analysis}

All Data are reported as mean \pm standard deviation (SD), unless otherwise specified. Significance was analyzed by student's $\mathrm{t}$ test using GraphPad Prism version 5.00 (GraphPad, San Diego, CA, USA) (* $p<0.05$; * $p<0.01$; $* * * p<0.001$; $* * * * 0<0.0001)$ unless otherwise specified.

\section{Results}

MINCR expression was increased in NSCLC tissues and cell lines

The expression of MINCR in clinical lung cancers, including LUAD and LUSC, was first identified using TCGA dataset. Results showed that MINCR expression 

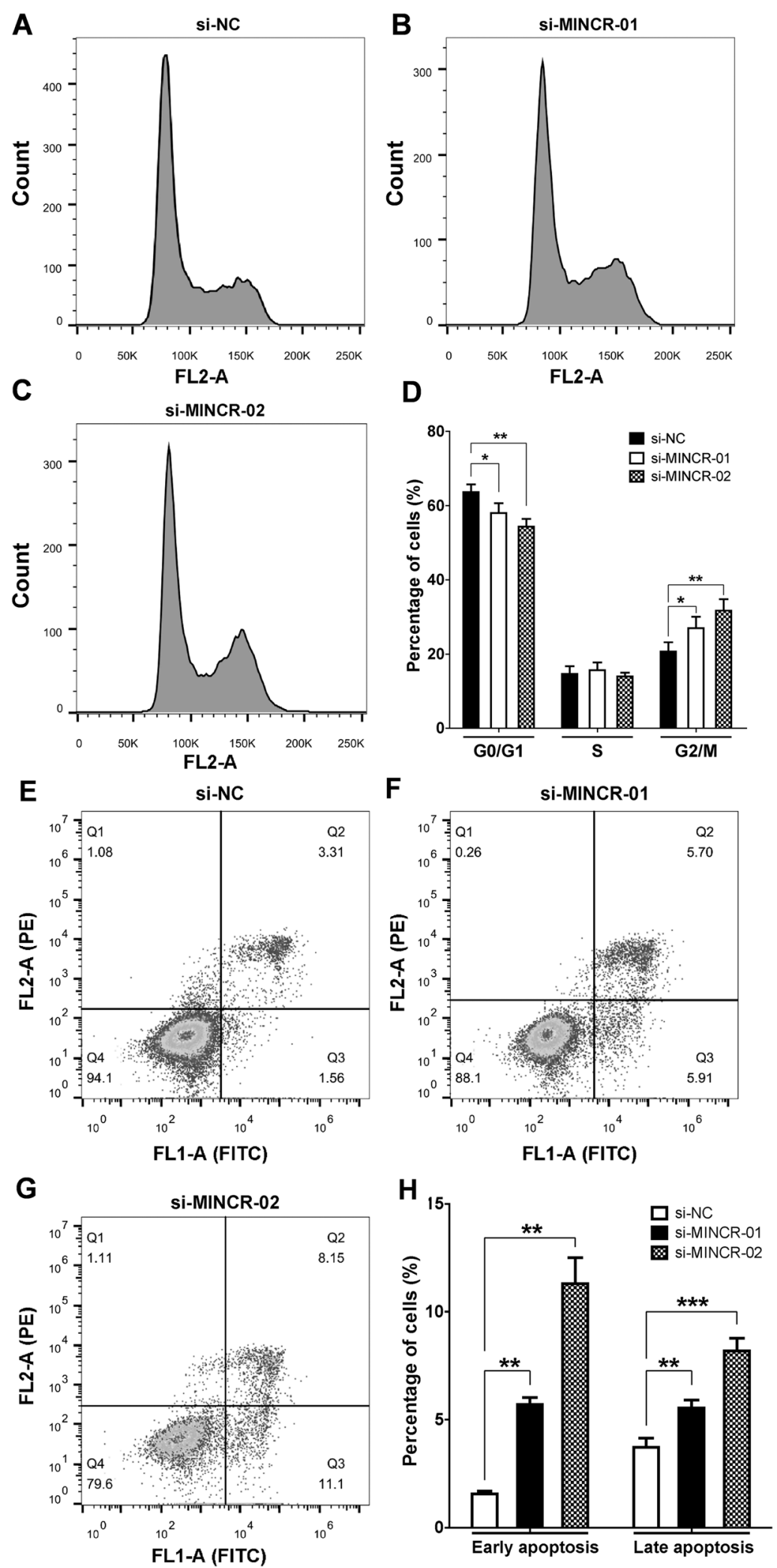

Fig. 3 (See legend on next page.) 
(See figure on previous page.)

Fig. 3 The effect of MINCR silencing on the cell cycle distribution and cell apoptosis of PC9 cells. a-c the representative figures of PC9 cells transfected with si-NC, si-MINCR-01, or si-MINCR-02, respectively. $\mathbf{d}$, the statistics of experiments (a-c). e-g the representative figures of PC9 cells transfected with si-NC, si-MINCR-01, and si-MINCR-02, respectively. $\mathbf{h}$, the statistics of experiments (e-g). Each experiment was repeated independently at least three times. Data were presented as mean $\pm \mathrm{SD} ;{ }^{*}, p<0.05 ;{ }^{*}, p<0.01 ;{ }^{* * *}, p<0.001$

was significantly increased in LUAD and LUSC samples compared with non-tumor controls (Fig. 1a and b). Furthermore, MINCR expression in the lung cancer and para-tumor tissues from 29 NSCLC patients was examined. As shown in Fig. 1c, MINCR expression was significantly increased in cancer tissues compared with para-tumor tissues. Next, the expression of MINCR in NSCLC cell lines (HCC827, A549, PC9) and normal control cell line $16 \mathrm{HBE}$ were detected. As expected, MINCR expression was highly expressed in two NSCLC cell lines, PC9 and A549, especially in PC9 (Fig. 1d). Then, PC9 and A549 were used in further studies.

\section{Silencing of MINCR significantly inhibited the proliferation of NSCLC cell lines}

SiRNAs targeting MINCR, including si-MINCR-01 and si-MINCR-02, were used to silence the expression of MINCR in PC9 and A549 cells. As shown in Fig. 2a, MINCR siRNAs efficiently decreased the expression of MINCR in PC9 cells compared with negative control siRNA. Furthermore, silencing of MINCR greatly inhibited cell viabilities and reduced the expression levels of Ki-67 (Fig. 2b-d), a biomarker for proliferation. Similar results were shown in MINCR-silenced A549 cells (Fig. 2e-h). These data indicated that silencing of MINCR suppressed the proliferation of NSCLC cells.

\section{Silencing of MINCR inhibited the proliferation of PC9 cells through inhibition of cell cycle and induction of apoptosis}

To further dissect the underlying mechanisms by which MINCR silencing inhibited growth of PC9 cells, flow-cytometer was used to analyze the proportions of cells in different cell cycle stages as well as the status of cell appoptosis. As shown in Fig. 3a-d, MINCR silencing significantly decreased G1/0 proportion and increased $G 2 / M$ proportion of PC9 cells. In addition, MINCR silencing in PC9 cells significantly induced apoptosis in these cells (Fig. 3e-h).

\section{Silencing of MINCR induced cell cycle arrest and apoptosis of NSCLC cells through decreasing c-Myc expression}

Since MINCR is a MYC-induced lncRNA, and has been reported to modulate c-Myc's transcriptional network in
Burkitt lymphoma cells, we sought to determine whether the function of MINCR may associated with the expression of c-Myc and its downstream genes [13]. Results showed that in PC9 cells treated with MINCR siRNA, cMyc expression was greatly decreased (Fig. 4a). Consistent with this, the expression of several down-stream targets, including cyclin A, cyclin D, CDK2 and CDK4, was significantly decreased after MINCR silencing (Fig. 4a and b). In addition, the expression of cell apoptosis-associated proteins also changed. As expected, the expression of Bcl-2 and Bax, two anti-apoptotic proteins, was decreased, while the expression of cleaved-PARP-1 seemed to be increased, in MINCR-silenced PC9 cells (Fig. 4c and d). In MINCR-silenced A549 cells, expression of c-Myc and proteins associated with cell cycle and apoptosis were also significantly changed (Fig. 4e-h).

\section{Over-expression of MINCR promoted the proliferation of PC9 cells through increasing c-Myc expression}

To increase the expression of MINCR, MINCR over-expression vector was constructed and then transfected into PC9 cells (Fig. 5a). Results showed that over-expression of MINCR dramatically increased the viabilities and Ki-67 expression (Fig. 5b-d). Mechanism studies showed that in PC9 cells, over-expression of MINCR enhanced c-Myc expression, which in turn increased the expression of some c-Myc downstream effectors, including CDK2, CDK4, Cyclin A, Cyclin D, resulting in promotion of cell cycle progression (Fig. 5e and f). However, in A549 and 16HBE cells, although transfection of MINCR over-expression vector significantly increased the expression of MINCR, the viabilities of these cells were not changed (Additional file 1: Figure S1).

\section{Discussion}

Recent studies have showed that IncRNA MINCR plays oncogenic roles in cancers, such as gallbladder cancer and hepatocellular carcinoma $[14,15]$. However, its precise roles in NSCLC and the underlying mechanisms are not fully elucidated. In this study, we identified that MINCR was highly increased in NSCLC tissues and cell lines. Furthermore, silencing of MINCR greatly reduced cell growth by inducing cell cycle arrest and apoptosis of NSCLC cells, while over-expressing MINCR remarkably enhanced cell proliferation, part of which are consistent with a previous report by Wang et al. [24]. For the mechanism studies, Wang et al. suggested that MINCR/ miR-126/ solute carrier family 7 member 5 (SLC7A5) axis was involved in NSCLC progression [24]. 
A

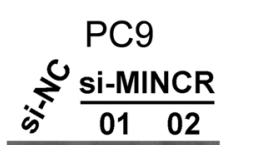

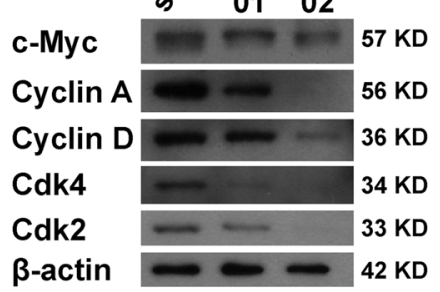

C

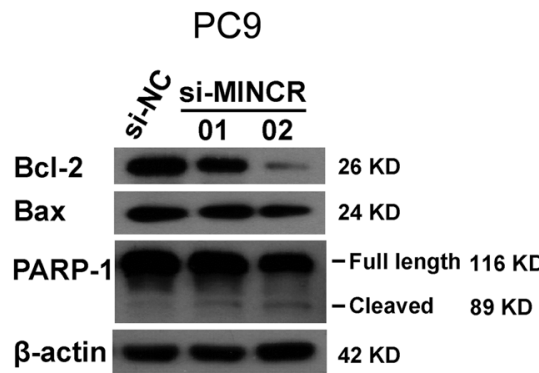

E

A549

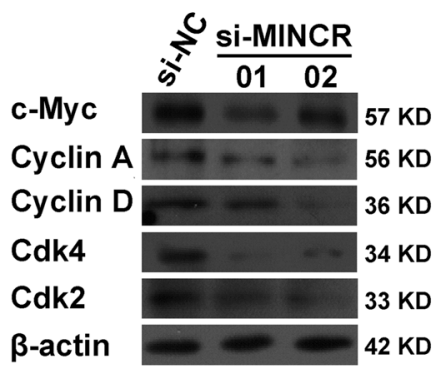

G

A549

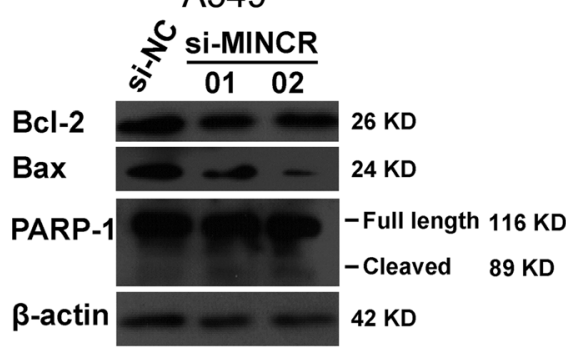

B

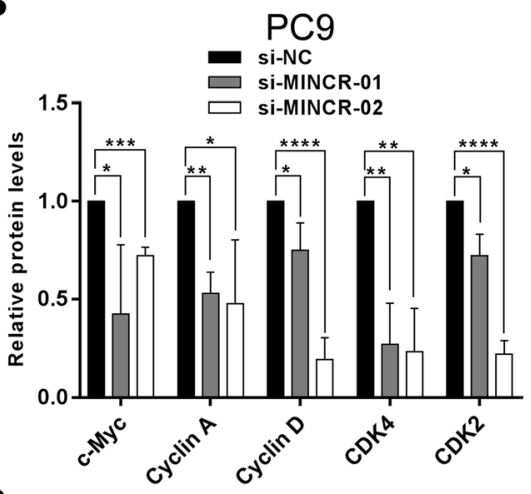

D

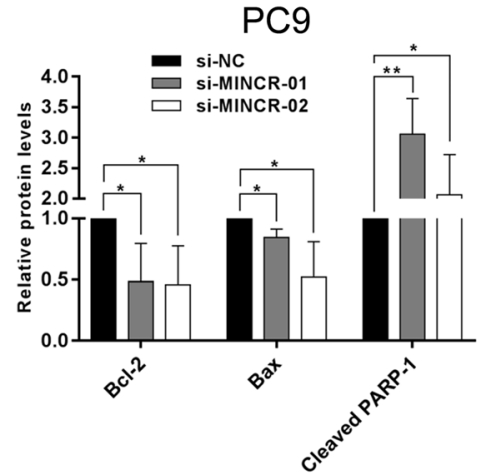

$\mathbf{F}$

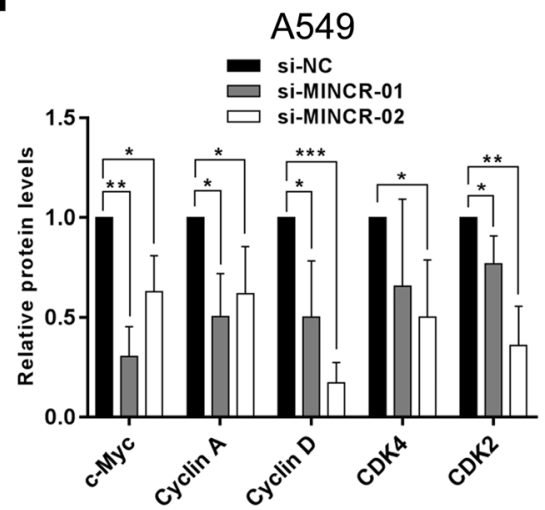

H

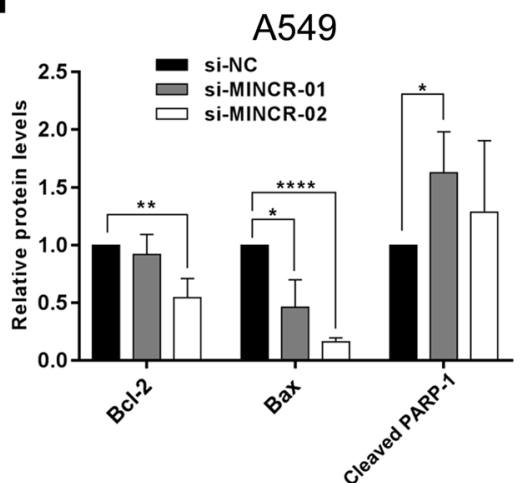


(See figure on previous page.)

Fig. 4 The effects of MINCR silencing on c-Myc and its downstream effectors. $\mathbf{a}-\mathbf{b}$, The protein expression levels (a) and statistic graphs (b) of cMyc and its downstream effectors Cyclin A, Cyclin D, CDK4, and CDK2 in PC9 cells with or without MINCR silencing. c-d, The protein expression levels (c) and statistic graphs (d) of BCl-2, Bax and PARP-1 (full length and cleaved forms) in PC9 cells with or without MINCR silencing. e-f, The protein expression levels (e) and statistic graphs (f) of c-Myc and its downstream effectors Cyclin A, Cyclin D, CDK4, and CDK2 in A549 cells with or without MINCR silencing. $\mathbf{g}-\mathbf{h}$, The protein expression levels ( $\mathbf{g}$ ) and statistic graphs (h) of Bcl-2, Bax and PARP-1 (full length and cleaved forms) in A549 cells with or without MINCR silencing. Each experiment was repeated independently at least three times. Data were presented as mean $\pm \mathrm{SD} ;{ }^{*}, p<0.05 ;{ }^{* *}, p<0.01{ }^{* * *}, p<0.001 ;{ }^{* * *}, p<0.0001$

Interestingly, our study elucidated a positive regulation of MINCR on c-Myc and its target genes, indicating a positive auto-regulatory loop between MINCR and c-Myc allowing their higher expression in NSCLC and accelerating NSCLC progression. Thus, MINCR may serve as a promising therapeutic target for the treatment of NSCLC.
C-Myc, a classical transcription factor, has been reported to regulate the expression of $15 \%$ of all human protein-coding genes, as well as some non-coding genes $[25,26]$. It was found to be greatly increased in different human cancers, and played pivotal roles in cancer progression [27]. Since MINCR was reported to be a modulator of the $\mathrm{c}-\mathrm{Myc}$ transcriptional network in Burkitt
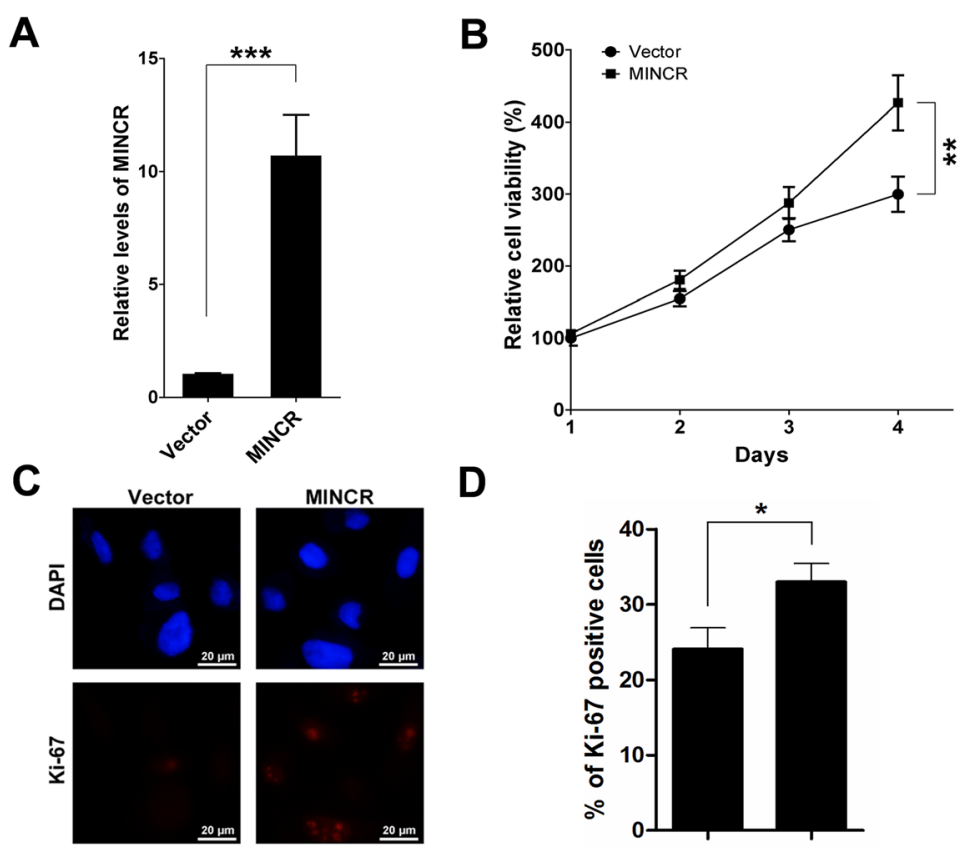

D
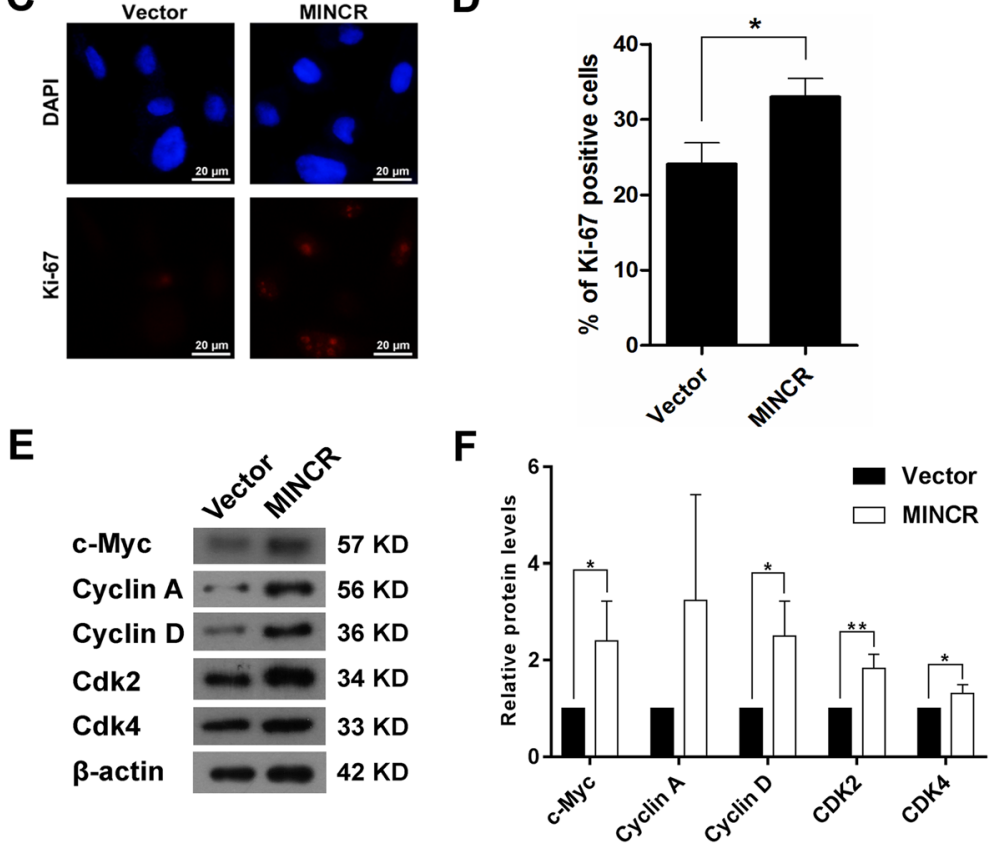

Fig. 5 The effect of MINCR over-expression on the proliferation of PC9 cells. a,Over-expression of MINCR in PC9 cells using MINCR overexpression vector. $\mathbf{b}-\mathbf{d}$, the corresponding cell viabilities of PC9 cells (b), representative images (c) and statistic graphs (d) of proliferation marker Ki-67 positive cells. e-f, The protein expression levels (e) and statistic graphs (f) of c-Myc and its downstream effectors Cyclin A, Cyclin D, CDK4, and CDK2 in PC9 cells with or without MINCR over-expression. Each experiment was repeated independently at least three times. Data were presented as mean $\pm \mathrm{SD} ;{ }^{*}, p<0.05 ;{ }^{* *}, p<0.01{ }^{* * *}, p<0.001$ 
lymphoma cells, we postulated that the function of MINCR in NSCLC may be associated with its regulation of c-Myc expression [13]. As expected, we found that silencing of MINCR reduced the expression of c-Myc and its target effectors, while over-expression of MINCR activated the expression of c-Myc and its target genes, indicating that MINCR was capable of promoting c-Myc transcription network in NSCLC cells. Further studies should explore the underlying mechanism of the regulation of c-Myc by MINCR in NSCLC.

Currently, several IncRNAs were reported to regulate cMyc at multiple levels, including transcription, translation, protein stability and activity, by interacting with different partners. For example, IncRNA colon cancer associated transcript 2 (CCAT2) promotes c-Myc transcription by strengthenning the binding of transcription factor transcription factor 7 like 2 (TCF7L2) to the c-Myc promoter in colon cancer; testis-associated highly-conserved oncogenic lncRNA (THOR) enhances c-Myc mRNA stability by interacting with insulin like growth factor 2 mRNA binding proteins (IGF2BPs) and facilitating its binding to c-Myc mRNA in skin squamous cell carcinoma; lncRNA prostate cancer associated transcript 1 (PCAT-1) stimulates c-Myc mRNA translation by competitively interacting with the 3 '-UTR of cMyc mRNA and prevent its binding with miR-34a-5p; whereas IncRNA protein disulfide isomerase family A member 3 pseudogene (PDIA3P) promotes c-Myc activities by increasing the occupancy of $\mathrm{c}-\mathrm{Myc}$ at the promoter of its target gene glucose-6-phosphate dehydrogenase (G6PD) in multiple myeloma cells [28-31]. In our current study, accompanied with the modulation of MINCR on c-Myc, the expression of c-Myc target genes exihibited the same trend, which is similar with the results from Doose's studies in Burkit lymphoma cells [13]. In following investigations, the direct or indirect interaction between MINCR and c-Myc needs to be studied, and whether MINCR promotes c-Myc by enhancing the binding of c-Myc to the promoters of its target effectors need to be determined. Besides, the relationship of MINCR and c-Myc need to be further assured in vivo.

\section{Conclusions}

Taken together, the current study indicated that MINCR as a regulator in NSCLC to enhance NSCLC progression by effectively inhibiting cell cycle arrest and apoptosis via increasing expression of c-Myc and its target effectors, which sheds light on the possible application of silencing of MINCR for the treatment of NSCLC.

\section{Additional file}

Additional file 1: Figure S1. The effect of MINCR over-expression on the viability of A549 and 16HBE cells. (A-B), Over-expression of MINCR in A549 cells using MINCR over-expression vector (A), and the

corresponding cell viabilities of A549 cells (B). (C-D), Over-expression of
MINCR in $16 \mathrm{HBE}$ cells using MINCR over-expression vector (C), and the corresponding cell viabilities of A549 cells (D). Each experiment was repeated independently at least three times. Data were presented as mean \pm SD; ***, $p<0.001$. (TIF $3238 \mathrm{~kb}$ )

\section{Abbreviations}

CDK: cylin-dependent kinase; EZH2: enhancer of zeste homolog 2; InCRNA: Long non-coding RNA; LUAD: lung adenocarcinoma; LUSC: lung squamous carcinoma; MINCR: MYC-induced long non-coding RNA; NSCLC: Non-small cell lung cancer; siRNA: small interfering RNA; TCGA: The Cancer Genome Atlas

\section{Acknowledgements}

Not applicable.

\section{Authors' contributions}

SC and TG carried out experiments, prepared figures, and wrote the manuscript. $Z \mathrm{~L}, \mathrm{LQ}, \mathrm{GX}, \mathrm{XZ}, \mathrm{FL}, \mathrm{HY}$, and $\mathrm{GL}$ were involved in the experiments, performed the statistical analysis, and wrote the manuscript. $\mathrm{HL}$ contributed to the conceptual design of the investigations, drafting and critically revising the remanuscript. All authors read and approved the final manuscript.

\section{Funding}

This work was supported by the National Natural Science Foundation of China (81672582); Natural Science Foundation of Jiangsu Province for Distinguished Young Scholars (BK20160013); Top Talent of Innovative Research Team of Jiangsu Province; Six Talent Peak Project from Government of Jiangsu Province (2015-SWYY-019); Senior Talent Start-up Funds of Jiangsu University (14JDG050); the 13th Batch of Planned Science and Technology Projects in 2011 of Zhenjiang (SH201 1052); Jiangsu Provincial Administration of Traditional Chinese Medicine (YB2017101); and Startup Fund for Distinguished Scholars, Affiliated Hospital of Jiangsu (jdfyRC2017002).

\section{Availability of data and materials}

All data generated or analyzed during this study are available from the corresponding author on reasonable request.

Ethics approval and consent to participate

This study was approved by the Human Research Ethics Committee of Jiangsu Univrsity.

\section{Consent for publication}

Informed consent was signed by all the patients.

\section{Competing interests}

The authors declare that they have no competing interests.

\section{Author details}

${ }^{1}$ Department of Cardiothoracic Surgery, Affiliated Hospital of Jiangsu University, Zhenjiang 212001, Jiangsu Province, China. ${ }^{2}$ School of Medicine, Jiangsu University, Zhenjiang 212013, Jiangsu Province, China. ${ }^{3}$ School of Pharmacy, Jiangsu University, Zhenjiang 212013, Jiangsu Province, China. ${ }^{4}$ Institute of Life Sciences, Jiangsu University, Zhenjiang 212013, Jiangsu Province, China.

Received: 26 March 2019 Accepted: 28 August 2019

Published online: 03 September 2019

References

1. Mortazavi A, Williams BA, McCue K, Schaeffer L, Wold B. Mapping and quantifying mammalian transcriptomes by RNA-Seq. Nat Methods. 2008;5(7):621-8.

2. Trapnell C, Williams BA, Pertea G, Mortazavi A, Kwan G, van Baren MJ, et al. Transcript assembly and quantification by RNA-Seq reveals unannotated transcripts and isoform switching during cell differentiation. Nat Biotechnol. 2010;28(5):511-5.

3. Cabili MN, Trapnell C, Goff L, Koziol M, Tazon-Vega B, Regev A, et al. Integrative annotation of human large intergenic noncoding RNAs reveals global properties and specific subclasses. Genes Dev. 2011;25(18):1915-27. 
4. Derrien $T$, Johnson R, Bussotti G, Tanzer A, Djebali S, Tilgner $H$, et al. The GENCODE v7 catalog of human long noncoding RNAs: analysis of their gene structure, evolution, and expression. Genome Res. 2012;22(9):1775-89.

5. Landers M, Calciano MA, Colosi D, Glatt-Deeley H, Wagstaff J, Lalande M. Maternal disruption of Ube3a leads to increased expression of Ube3a-ATS in trans. Nucleic Acids Res. 2005:33(13):3976-84.

6. Fu X, Ravindranath L, Tran N, Petrovics G, Srivastava S. Regulation of apoptosis by a prostate-specific and prostate cancer-associated noncoding gene, PCGEM1. DNA Cell Biol. 2006;25(3):135-41.

7. Guttman M, Rinn JL. Modular regulatory principles of large non-coding RNAs. Nature. 2012;482(7385):339-46.

8. Rinn JL, Chang HY. Genome regulation by long noncoding RNAs. Annu Rev Biochem. 2012;81:145-66.

9. Kotake Y, Nakagawa T, Kitagawa K, Suzuki S, Liu N, Kitagawa M, et al. Long non-coding RNA ANRIL is required for the PRC2 recruitment to and silencing of p15(INK4B) tumor suppressor gene. Oncogene. 2011;30(16):1956-62.

10. Kim T, Cui R, Jeon YJ, Lee JH, Sim H, Park JK, et al. Long-range interaction and correlation between MYC enhancer and oncogenic long noncoding RNA CARLo-5. Proc Natl Acad Sci U S A. 2014:111(11):4173-8.

11. Hu X, Feng Y, Zhang D, Zhao SD, Hu Z, Greshock J, et al. A functional genomic approach identifies FAL1 as an oncogenic long noncoding RNA that associates with BMI1 and represses p21 expression in cancer. Cancer Cell. 2014;26(3):344-57.

12. Yuan JH, Yang F, Wang F, Ma JZ, Guo YJ, Tao QF, et al. A long noncoding RNA activated by TGF-beta promotes the invasion-metastasis cascade in hepatocellular carcinoma. Cancer Cell. 2014;25(5):666-81.

13. Doose G, Haake A, Bernhart SH, Lopez C, Duggimpudi S, Wojciech F, et al. MINCR is a MYC-induced IncRNA able to modulate MYC's transcriptional network in Burkitt lymphoma cells. Proc Natl Acad Sci U S A. 2015;112(38):E5261-70.

14. Wang SH, Yang Y, Wu XC, Zhang MD, Weng MZ, Zhou D, et al. Long noncoding RNA MINCR promotes gallbladder cancer progression through stimulating EZH2 expression. Cancer Lett. 2016;380(1):122-33.

15. Cao J, Zhang D, Zeng L, Liu F. Long noncoding RNA MINCR regulates cellular proliferation, migration, and invasion in hepatocellular carcinoma. Biomed Pharmacother. 2018;102:102-6.

16. Wang H, Gutierrez-Uzquiza A, Garg R, Barrio-Real L, Abera MB, Lopez-Haber $C$, et al. Transcriptional regulation of oncogenic protein kinase C (PKC) by STAT1 and Sp1 proteins. J Biol Chem. 2014:289(28):19823-38.

17. Wang SH, Wu XC, Zhang MD, Weng MZ, Zhou D, Quan ZW. Long noncoding RNA H19 contributes to gallbladder cancer cell proliferation by modulated miR-194-5p targeting AKT2. Tumour Biol. 2016;37(7):9721-30.

18. Liu H, Xiao F, Serebriiskii IG, O'Brien SW, Maglaty MA, Astsaturov I, et al. Network analysis identifies an HSP90-central hub susceptible in ovarian cancer. Clin Cancer Res. 2013;19(18):5053-67.

19. Liu H, Lu J, Hua Y, Zhang P, Liang Z, Ruan L, et al. Targeting heat-shock protein 90 with ganetespib for molecularly targeted therapy of gastric cancer. Cell Death Dis. 2015;6:e1595.

20. Tu Z, Aird KM, Bitler BG, Nicodemus JP, Beeharry N, Xia B, et al. Oncogenic Ras regulates BRIP1 expression to induce dissociation of BRCA1 from chromatin, inhibit DNA repair, and promote senescence. Dev Cell. 2011; 21(6):1077-91.

21. Tu Z, Zhuang $X$, Yao $Y$, Zhang R. BRG1 is required for formation of senescence-associated heterochromatin foci induced by oncogenic RAS or BRCA1 loss. Mol Cell Biol. 2013;33(9):1819-29.

22. Chauvin L, Goupille C, Blanc C, Pinault M, Domingo I, Guimaraes C, et al. Long chain n-3 polyunsaturated fatty acids increase the efficacy of docetaxel in mammary cancer cells by downregulating Akt and PKCepsilon/ delta-induced ERK pathways. Biochim Biophys Acta. 2016;1861(4):380-90.

23. Shang D, Wu Y, Ding Y, Lu Z, Shen Y, Zhu F, et al. Identification of a pyridine derivative inducing senescence in ovarian cancer cell lines via P21 activation. Clin Exp Pharmacol Physiol. 2018:45(5):452-60.

24. Wang J, Ding $M$, Zhu H, Cao Y, Zhao W. Up-regulation of long noncoding RNA MINCR promotes non-small cell of lung cancer growth by negatively regulating miR-126/SLC7A5 axis. Biochem Biophys Res Commun. 2019; 508(3):780-4.

25. Li Z, Van Calcar S, Qu C, Cavenee WK, Zhang MQ, Ren B. A global transcriptional regulatory role for C-Myc in Burkitt's lymphoma cells. Proc Natl Acad Sci U S A. 2003;100(14):8164-9.

26. Winkle M, van den Berg A, Tayari M, Sietzema J, Terpstra M, Kortman G, et al. Long noncoding RNAs as a novel component of the Myc transcriptional network. FASEB J. 2015;29(6):2338-46.
27. Kalkat M, De Melo J, Hickman KA, Lourenco C, Redel C, Resetca D, et al. MYC deregulation in primary human cancers. Genes (Basel). 2017;8(6):151.

28. Ling H, Spizzo R, Atlasi Y, Nicoloso M, Shimizu M, Redis RS, et al. CCAT2, a novel noncoding RNA mapping to 8q24, underlies metastatic progression and chromosomal instability in colon cancer. Genome Res. 2013;23(9):1446-61.

29. Liu Z, Wu G, Lin C, Guo H, Xu J, Zhao T. IGF2BP1 over-expression in skin squamous cell carcinoma cells is essential for cell growth. Biochem Biophys Res Commun. 2018;501(3):731-8.

30. Prensner JR, Chen W, Han S, lyer MK, Cao Q, Kothari V, et al. The long noncoding RNA PCAT-1 promotes prostate cancer cell proliferation through cMyc. Neoplasia. 2014;16(11):900-8

31. Yang $X, Y e ~ H$, He M, Zhou X, Sun N, Guo W, et al. LncRNA PDIA3P interacts with cMyc to regulate cell proliferation via induction of pentose phosphate pathway in multiple myeloma. Biochem Biophys Res Commun. 2018;498(1):207-13.

\section{Publisher's Note}

Springer Nature remains neutral with regard to jurisdictional claims in published maps and institutional affiliations.
Ready to submit your research? Choose BMC and benefit from:

- fast, convenient online submission

- thorough peer review by experienced researchers in your field

- rapid publication on acceptance

- support for research data, including large and complex data types

- gold Open Access which fosters wider collaboration and increased citations

- maximum visibility for your research: over $100 \mathrm{M}$ website views per year

At $\mathrm{BMC}$, research is always in progress.

Learn more biomedcentral.com/submissions 